\title{
Cyanide in bronchoalveolar lavage is not diagnostic for Pseudomonas aeruginosa in children with cystic fibrosis
}

\author{
M.D. Stutz*, , C.L. Gangell*, L.J. Berry*, L.W. Garratt*, B. Sheil ${ }^{*,+}$ and P.D. Sly*, ${ }^{\star},+$ \\ on behalf of the Australian Respiratory Early Surveillance Team for Cystic Fibrosis \\ (AREST CF) $^{\S}$
}

ABSTRACT: Early detection of the cyanobacterium Pseudomonas aeruginosa in the lungs of young children with cystic fibrosis (CF) is considered the key to delaying chronic pulmonary disease. We investigated whether cyanide in bronchoalveolar lavage (BAL) fluid could be used as an early diagnostic biomarker of infection.

Cyanide was measured in 226 BAL samples (36 P. aeruginosa infected) obtained from 96 infants and young children with CF participating in an early surveillance programme involving annual BAL.

Cyanide was detected in $\mathbf{9 7 . 2 \%}$ of $\boldsymbol{P}$. aeruginosa infected and $\mathbf{6 0 . 5 \%}$ of uninfected samples. Cyanide concentrations were significantly higher in BALs infected with $P$. aeruginosa (median (25th-75th percentile) $27.3(22.1-33.3) \mu \mathrm{M})$ than those which were not (17.2 (7.85-23.0) $\mu \mathrm{M}$, $\mathbf{p}<\mathbf{0 . 0 0 1 )}$. The best sensitivity, specificity, positive and negative predictive values were obtained with a cut-off concentration of $20.6 \mu \mathrm{M}$, and were $83 \%, 66 \%, 32 \%$ and $96 \%$, respectively. Neutrophil number in BAL was a significant predictor of cyanide concentration $(p<0.001)$.

Cyanide concentration can distinguish between $P$. aeruginosa infected and uninfected BALs as a group, but not individually; therefore, cyanide is a poor diagnostic biomarker of $\boldsymbol{P}$. aeruginosa infection. Cyanide levels in BAL are related to the level of neutrophilic inflammation.

KEYWORDS: Biomarker, cyanide, cystic fibrosis, Pseudomonas aeruginosa

hronic pulmonary infection with Pseudomonas aeruginosa is an important cause of morbidity and mortality in patients with cystic fibrosis (CF). The acquisition of this opportunistic pathogen is associated with significantly worse lung function and a poorer prognosis [1-3]. Recent data from our bronchoalveolar lavage (BAL)-based surveillance programme showed a median age of acquisition of pulmonary infection with $P$. aeruginosa of $\sim 26$ months in infants diagnosed with $C F$, following detection by newborn screening [4]. The majority of these infections occur in the absence of clinically apparent lung disease and are amenable to successful eradication for periods of up to $5 \mathrm{yrs}$ [4]. However, around $80 \%$ of patients eventually develop chronic infections, which are virtually impossible to eliminate using current treatments [5]. Consequently, several paediatric $\mathrm{CF}$ clinics have initiated respiratory disease surveillance programmes which aim to detect and treat $P$. aeruginosa infections early, thereby preventing or delaying the chronic phase.

The current "gold standard" for diagnosing $P$. aeruginosa in infants and young children with $\mathrm{CF}$ is conventional bacterial culture of BAL fluid [6]. Unfortunately, such techniques lack sensitivity [7]. The low density of $P$. aeruginosa typically present in the early stages of lung infection may, therefore, be particularly difficult to diagnose by culture. More sensitive diagnostic assays are required if the goal of early eradication of $P$. aeruginosa is to be fully realised.

$P$. aeruginosa is a member of a small group of bacteria which are capable of generating hydrogen cyanide $(\mathrm{HCN})$ [8]. Cyanide is produced exclusively under microaerobic conditions [9],
AFFILIATIONS

*Division of Clinical Sciences, Telethon Institute for Child Health Research and Centre for Child Health Research, University of Western Australia.

${ }^{*}$ School of Biological Sciences and Biotechnology, Faculty of Sustainability, Environmental and Life Sciences, Murdoch University and 'Dept of Respiratory Medicine, Princess Margaret Hospital for Children, Perth, Australia. +These authors shared senior author responsibilities

${ }^{\S} \mathrm{A}$ full list of the AREST CF team members and their affiliations can be found in the Acknowledgements section.

CORRESPONDENCE

P.D. Sly

Queensland Children's Medical Research Institute, University of Queensland, Level 4, Foundation Building Royal Children's Hospital Herston Qld Australia

E-mail: p.sly@uq.edu.au

Received:

Feb 122010

Accepted after revision:

May 302010

First published online: June 182010 
which are believed to exist in the thick mucus layers in the $\mathrm{CF}$ lung [10]. Given that $P$. aeruginosa is the only cyanogenic pathogen frequently isolated from young children with $\mathrm{CF}$, the measurement of cyanide in the lungs may offer a cultureindependent approach for detecting the infection in these patients. However, whether the necessary conditions for cyanide production are present in young children without chronic infection is debatable. The measurement of cyanide in breath as a diagnostic assay for $P$. aeruginosa is attracting research interest [11]. Although preliminary data show promise, breath analysis requires highly sophisticated and expensive instruments. Other studies have reported the detection of cyanide in sputum using a simple and inexpensive potentiometric assay $[12,13]$. Detectable cyanide was found in most of the $P$. aeruginosa infected patients who were studied. In contrast, cyanide was only detected in one uninfected control patient who, intriguingly, was diagnosed with the infection shortly after.

The aim of our study was to determine whether cyanide was detectable in BAL from $P$. aeruginosa infected children with $\mathrm{CF}$, and whether its detection could be used as an early diagnostic aid for $P$. aeruginosa.

\section{MATERIALS AND METHODS}

A more detailed description of the methods used in the collection and analysis of BAL has been published previously [14].

\section{$B A L$}

BAL samples were obtained from infants and young children with CF who participated in the early respiratory disease surveillance programme operated by the Australian Respiratory Early Surveillance Team for Cystic Fibrosis (AREST CF) [15]. This programme operates in the paediatric CF clinics at Princess Margaret Hospital for Children (Perth, Australia) and Royal Children's Hospital (Melbourne, Australia). The surveillance programme was approved by the ethics committee at each institution, and parental consent was obtained separately for each aspect.

Two study populations were used: 1) a cross-sectional population consisting of as many BAL samples as were available in the biobank; and 2) a longitudinal population consisting of BAL samples from children who had been infected with $P$. aeruginosa and from whom BAL samples were available 12 months prior to infection, or 3 or 12 months after undergoing a specific eradication programme [4].

The programme includes annual bronchoscopy and BAL performed under general anaesthesia. Three aliquots of normal saline $\left(1 \mathrm{~mL} \cdot \mathrm{kg}^{-1}\right)$ were instilled into the right middle lobe and retrieved using low-pressure suction. The first aliquot was submitted for microbiological assessment. The remaining aliquots were combined and stored on ice until processing in the laboratory (within $3 \mathrm{~h}$ ). A single aliquot was also obtained from the lingula or most affected lobe on chest computed tomography and sent for microbiological assessment. BAL fluid was centrifuged (300xg for $5 \mathrm{~min}$ ) and the supernatant decanted, aliquoted and stored at $-80^{\circ} \mathrm{C}$ for subsequent analysis of inflammatory markers. Cell pellets were resuspended to perform total and differential cell counts.

\section{Microbiology}

Bacteria and fungi were identified by culture, and viruses detected by direct immunofluorescence and/or rapid culture. Microbial densities $<10^{4} \mathrm{CFU} \cdot \mathrm{mL}^{-1}$ were recorded as isolated colonies. BAL fluid containing only mixed oral flora or isolated colonies was classified as uninfected for the purposes of this study. However, to reflect current clinical practice [4] the presence of $P$. aeruginosa at any density was regarded as an infection, as was the presence of viruses, which were not quantified. $P$. aeruginosa isolates but were classified as mucoid or nonmucoid depending on their colony morphology on blood agar. Colonies were not classified when the morphology was ambiguous.

\section{Instrumentation and calibration}

Cyanide was assayed using a uniPROBE cyanide ion-selective electrode (ISE) connected to a smartCHEM-Ion meter (TPS, Springwood, QLD, Australia). The ISE was washed and rinsed with deionised water and blotted dry between measurements. Calibration was performed before each use with $100 \mu \mathrm{M}$ and $10^{4} \mu \mathrm{M}$ solutions of standard cyanide (Fluka, Castle Hill, NSW, Australia) prepared in $0.06 \mathrm{M} \mathrm{NaOH}$ (Merck, Kilsyth, VIC, Australia). After conditioning for $\sim 30 \mathrm{~min}$ in deionised water, the ISE was recalibrated. A standard curve was then constructed using 5, 15, 25, 50 and $100 \mu \mathrm{M}$ solutions of cyanide in order to check the precision of the two-point calibration.

\section{Cyanide measurement}

$400 \mu \mathrm{L}$ of BAL fluid was added to $800 \mu \mathrm{L}$ of $0.06 \mathrm{M} \mathrm{NaOH}$. This trapped the cyanide in solution, raised the $\mathrm{pH}$ of the sample to within the working range of the ISE, and minimised the volume of BAL fluid required to submerge the ISE tip. BAL fluid was measured in triplicate and the results were averaged. The investigator was blinded to the patient clinical data. Preliminary "spike-and-recovery" experiments were undertaken to assess the accuracy of the ISE for measuring cyanide in BAL fluid and a correction equation derived (see supplementary material for details). All cyanide measurements were adjusted using the following equation and corrected for dilution.

$$
\text { actual }[\text { cyanide] }=0.576(\text { measured }[\text { cyanide] })+2.36
$$

\section{Statistics}

Data are presented as either the median (25th-75th percentile) or mean $\pm \mathrm{SD}$, unless otherwise stated. Statistical analyses requiring clustered robust standard errors (CRSE) were performed using Stata v10.0 (StataCorp, College Station, TX, USA). Graphs and all other statistics were prepared using SigmaPlot v11.0 and SigmaStat v3.5 (Systat Software Inc., San Jose, CA, USA), respectively. A two-tailed p-value of $<0.05$ was considered statistically significant.

\section{Cross-sectional population}

Cyanide concentrations were compared between two groups using unpaired t-tests. Least-squares linear regression was used to relate cyanide level with bacterial density. Both analyses involved CRSE to account for multiple BALs from the same children. Cyanide concentrations were compared between three groups (uninfected, infected (non- $P$. aeruginosa) and infected with $P$. aeruginosa) by mixed-effects linear regression. Multiple linear regression examined associations 
between inflammatory markers and cyanide concentration. Inflammatory marker data were $\log _{10}$-transformed before analysis.

\section{Longitudinal population}

Samples from 15 children in whom infection with $P$. aeruginosa was detected and in whom BAL samples were available 12 months prior to infection $(n=15)$, or in whom BAL samples were available 3 months post-eradication $(n=12)$, or 12 months post-eradication $(n=10)$ were compared using paired t-tests without CRSE as follows: 12 months pre-compared with time of infection; time of infection compared with 3 months posteradication; or time of infection compared with 12 months post-eradication.

\section{RESULTS}

\section{Cyanide is detectable in BAL}

226 BALs from 96 children with CF (54\% male) were analysed for cyanide (table 1). Cyanide levels ranged from below the detection limit to $58.9 \mu \mathrm{M}$, with 150 out of 226 BALs $(66.4 \%)$ containing detectable levels. The median concentration among those with detectable cyanide was 22.9 (19.1-29.7) $\mu \mathrm{M}$. The group mean coefficient of variation for cyanide measurements was $8.22 \pm 4.84 \%$.

\section{Cyanide is associated with $\mathrm{P}$. aeruginosa infection}

The median cyanide concentration was significantly higher in the $P$. aeruginosa infected group of BALs compared with those not infected with the pathogen (table 1). The percentage of BALs containing detectable cyanide was also higher in the $P$. aeruginosa infected group (table 1 ).

The density of $P$. aeruginosa in BAL ranged from $10^{3}$ to $10^{7} \mathrm{CFU} \cdot \mathrm{mL}^{-1}$. However, no significant relationship was observed between density and cyanide concentration in BAL $\left(\mathrm{r}^{2}=0.0297, \mathrm{p}=0.23\right)$.

The $P$. aeruginosa phenotype was specified for 28 out of the 36 infected samples. Cyanide levels were higher with mucoid strains $(n=11,35.3 \pm 13.0 \mu \mathrm{M})$ than nonmucoid strains $(n=17$, $28.4 \pm 7.10 \mu \mathrm{M})$, although the difference was not significant $(\mathrm{p}=0.15)$.

The analyses reported previously were repeated using only the first BAL obtained from each child. While the numbers were smaller, including those infected with $P$. aeruginosa, the pattern of the results was essentially unchanged (table E1 in the supplementary material).

\section{Longitudinal changes in cyanide concentration}

BALs were available from 15 children at the time of their first $P$. aeruginosa diagnosis, as well as 12 months prior (mean \pm SD $11 \pm 1.9$ months). Eight (53\%) patients showed an increase in cyanide concentration at the time of diagnosis of $P$. aeruginosa infection compared to 12 months prior, while a decrease was observed in the remaining seven patients (fig. 1a). The group mean cyanide concentration 12 months prior to diagnosis $(26.6 \pm 12.2 \mu \mathrm{M})$ was not significantly different to the concentration at the time of diagnosis $(29.6 \pm 9.59 \mu \mathrm{M}, \mathrm{p}=0.50)$.

Cyanide concentrations at the time of infection with $P$. aeruginosa were also compared with those at two time intervals following eradication therapy: the 3 month follow-up BAL (4.0 \pm 0.84 months later, $n=12)$ and the following annual BAL $(12 \pm 1.3$ months later; $\mathrm{n}=10) .3$ months post-eradication a decrease in cyanide concentration was observed in seven (58\%) out of 12 cases, with the remaining five cases showing an increase (fig. 1b). Decreases in cyanide concentration were observed in all cases 12 months post-eradication (fig. 1b). Levels decreased from a mean of $26.2 \pm 4.93 \mu \mathrm{M}$ to $16.0 \pm$ $6.66 \mu \mathrm{M}(\mathrm{p}<0.001)$

\section{Cyanide has poor predictive value}

The sensitivity, specificity, positive predictive value (PPV) and negative predictive value for the presence of cyanide as a diagnostic indicator of $P$. aeruginosa infection are shown in table 2. Microbiology data were used as the reference standard and the presence of any detectable cyanide $(\geqslant 15.7 \mu \mathrm{M})$ was regarded as a positive result. PPV was notably very low. We therefore considered the possibility that higher cut-off cyanide concentrations may yield more desirable diagnostic parameters. The concentrations which gave the best compromise between sensitivity and specificity were $18.8 \mu \mathrm{M}$ and $20.6 \mu \mathrm{M}$ (table 2). However, increasing the cut-off concentration only marginally improved the PPV.

\section{The presence of other pathogens affects cyanide concentration}

To determine the effect of other microorganisms in BAL on cyanide concentration, all 226 BALs were classified into three groups based on microbiological data. Uninfected samples

\section{TABLE 1 Demographic characteristics of the study population}

Infection status

\begin{tabular}{ccc}
\hline $\boldsymbol{P}$. aeruginosa infected & $\boldsymbol{P .}$ aeruginosa uninfected & p-value \\
36 & 190 & \\
$17(47)$ & $103(54)$ & 0.56 \\
$3.5(0.55-9.9)$ & $3.0(0.13-8.2)$ & 0.32 \\
$35(97)$ & $115(61)$ & $<0.001$ \\
$27.3(22.1-33.3)$ & $17.2(7.85-23.0)$ &
\end{tabular}

Data are presented as median (25th-75th percentile) or $n$ (\%), unless otherwise stated. Data for age is presented as median (range). P. aeruginosa: Pseudomonas aeruginosa; BAL: bronchoalveolar lavage. 


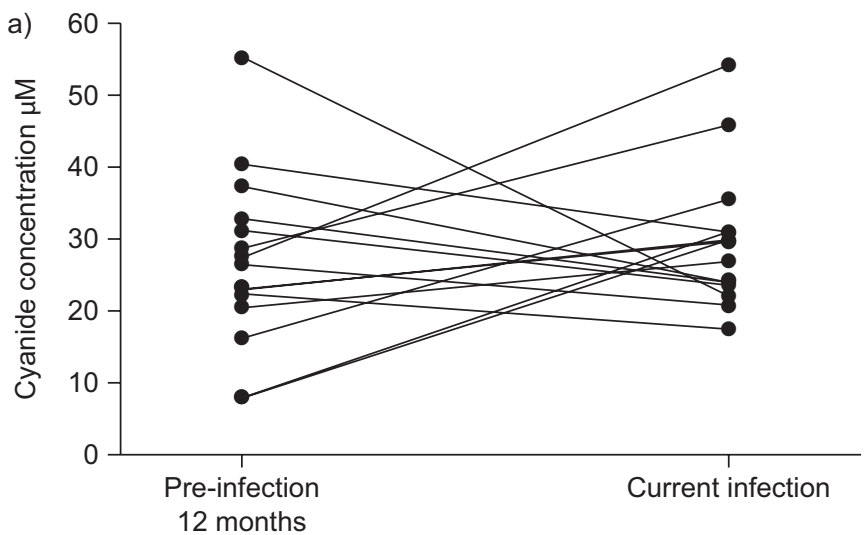

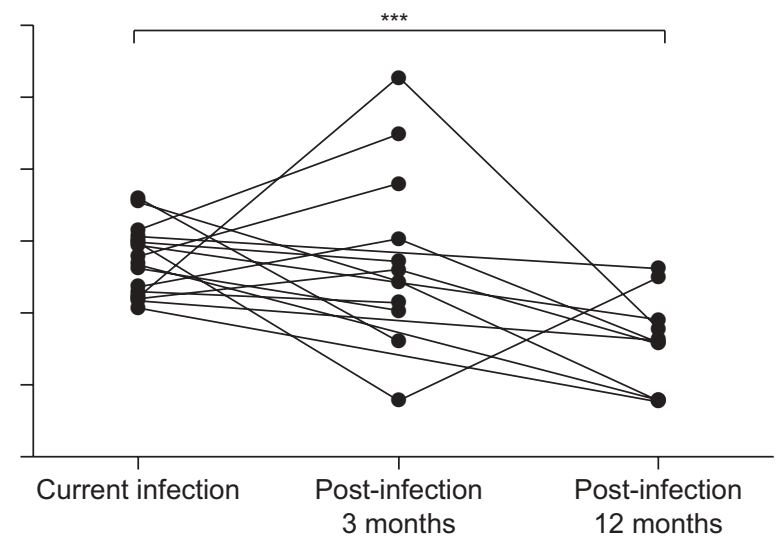

FIGURE 1. Longitudinal changes in cyanide concentration in bronchoalveolar lavage fluid before, during and after infection with Pseudomonas aeruginosa. Changes in concentration a) during infection compared with prior 12 months $(n=15)$, and b) 3 months $(n=12)$ and 12 months $(n=10)$ after eradication of infection. ${ }^{\star \star \star}: p<0.001$.

$(\mathrm{n}=151)$ had a median cyanide concentration of $17.0(7.85-$ 22.6) $\mu \mathrm{M}$. Samples infected with pathogens other than $P$. aeruginosa $(\mathrm{n}=39)$ had a slightly higher concentration $(18.2$ (7.85-26.9) $\mu \mathrm{M})$, although this was not significant (table 3). Those infected with $P$. aeruginosa $(n=36)$ had a significantly higher cyanide level than both other groups (27.3 (22.133.3) $\mu \mathrm{M}$ ) (table 3).

\section{Cyanide concentration is related to neutrophilic inflammation}

In multiple linear regression $(n=195)$, neutrophil number (cells $\cdot \mathrm{mL}^{-1}$ of BAL fluid retrieved from the lung) was a significant predictor of cyanide concentration in BAL $(\mathrm{p}<0.001)$. Higher neutrophil numbers corresponded to higher cyanide concentrations (partial regression coefficient $=6.72$ ). Macrophage number and interleukin-8 concentration were not significant confounding variables $(\mathrm{p}=0.701$ and 0.668 , respectively).

Neutrophil number was included as a covariate in the comparison of cyanide concentration between uninfected, infected (non- $P$. aeruginosa) and $P$. aeruginosa infected BALs. The only relationship which remained significant was that between uninfected samples and those infected with $P$. aeruginosa (table 3). Overall, the p-values increased.

\begin{tabular}{|c|c|c|c|c|}
\hline TABLE 2 & \multicolumn{4}{|c|}{$\begin{array}{l}\text { Diagnostic parameters of cyanide at differen } \\
\text { cut-off concentrations for Pseudomonas } \\
\text { aeruginosa infection }\end{array}$} \\
\hline \multirow{2}{*}{\multicolumn{2}{|c|}{ Diagnostic parameter }} & \multicolumn{3}{|c|}{ Cut-off cyanide concentration $\mu \mathrm{M}$} \\
\hline & & $15.7^{\#}$ & 18.8 & 20.6 \\
\hline \multicolumn{2}{|l|}{ Sensitivity \% } & 97.2 & 88.9 & 83.3 \\
\hline \multicolumn{2}{|l|}{ Specificity \% } & 39.5 & 57.4 & 66.3 \\
\hline \multicolumn{2}{|c|}{ PPV \% } & 23.3 & 28.3 & 31.9 \\
\hline \multicolumn{2}{|l|}{ NPV \% } & 98.7 & 96.5 & 95.5 \\
\hline
\end{tabular}

PPV: positive predictive value; NPV: negative predictive value. ${ }^{*}$ : the detection limit of the assay for cyanide.

\section{DISCUSSION}

Our results are consistent with previous studies that reported the detection of cyanide in the respiratory tract of $P$. aeruginosa infected patients with CF [11-13]. Our study demonstrated the presence of cyanide in two thirds of BALs from young children with CF. Cyanide was detectable both in the presence and absence of $P$. aeruginosa infection detected in BAL culture, although those infected with the pathogen had higher levels of cyanide overall. The diagnostic value of cyanide was poor, and increasing the cut-off concentration defining a positive result produced only a marginal improvement in PPV and sensitivity. Cyanide concentration correlated strongly with neutrophil number in BAL. Differences in neutrophil number explained most of the differences in cyanide concentration between the uninfected, infected (non- $P$. aeruginosa) and infected (with $P$. aeruginosa) groups. The difference between uninfected samples and those infected with $P$. aeruginosa could not be accounted for in this way.

In terms of diagnostic value, the presence of cyanide was a very sensitive indicator of $P$. aeruginosa infection, with almost all $P$. aeruginosa infected samples containing detectable cyanide. However, due to the large number of $P$. aeruginosa uninfected samples containing cyanide, specificity was very low. Cyanide is, therefore, not a reliable diagnostic biomarker of the presence of $P$. aeruginosa infection in the lungs of young children; rather it appears to be a marker of neutrophilic inflammation. One possible value of measuring cyanide would be to support a negative culture result, since the absence of cyanide was almost always associated with the absence of $P$. aeruginosa.

The presence of cyanide in a large proportion of BALs not infected with $P$. aeruginosa in this study is in contrast to previous reports. While the possibility that these samples returned a false-negative culture result cannot be eliminated, this would imply that the prevalence of $P$. aeruginosa in children is much higher than presently thought [4]. The presence of cyanide in BAL in the absence of $P$. aeruginosa intuitively suggests the existence of a non-Pseudomonal source. No child in the present study was infected with other known cyanogenic bacterium [16]. We therefore considered the possibility that cyanide levels may have been related to 


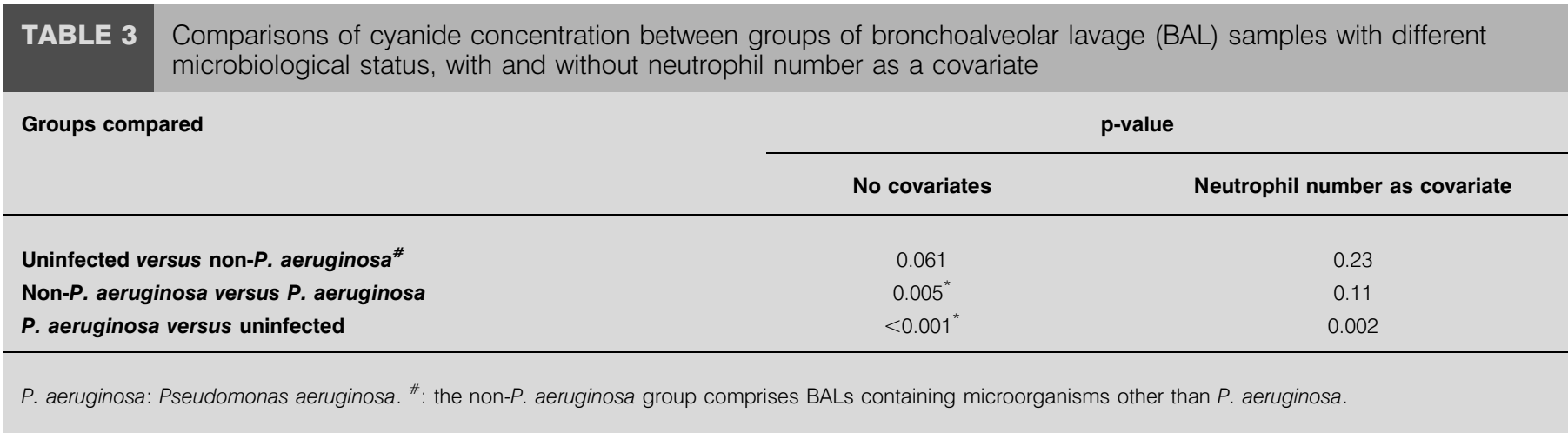

inflammatory responses in the lung, since the intensity of inflammation is significantly greater when pathogens are present, regardless of their identity [4, 17]. Inflammation also occurs in children with CF in the absence of detectable infections [14], which could explain the presence of cyanide in many of the uninfected samples. Our finding that neutrophil number was a significant predictor of cyanide concentration is consistent with several previous reports which suggest that neutrophil activity may be associated with cyanide generation. Early studies attributed $6-12 \%$ of the cyanide produced by neutrophils during phagocytosis to glycyl residues in bacterial peptidoglycan [18]. The neutrophilic enzyme myeloperoxidase (MPO) was previously shown to chlorinate glycine to an unstable intermediate, which subsequently decayed, producing HCN [19]. It was later discovered that most neutrophilderived cyanide was produced by the oxidation of thiocyanate $\left(\mathrm{SCN}^{-}\right)$to hypothiocyanite $\left(\mathrm{OSCN}^{-}\right)$by $\mathrm{MPO}$, with the latter ion hydrolysing to $\mathrm{HCN}[20,21] . \mathrm{SCN}^{-}$is ubiquitous in the body, and has been found in high concentrations $(0.46 \mathrm{mM})$ in undiluted human tracheobronchial secretions from intubated intensive care patients [22]. The generation of $\mathrm{OSCN}^{-}$, which has antimicrobial properties, is believed to constitute a host defence mechanism [23]. Neutrophil-mediated cyanide generation may therefore explain the results of our study, as well as those of STAMYR et al. [24], who detected low cyanide levels in the breath from healthy adults with no lung infections.

A limitation of the present study was the use of microbiological culture of BAL fluid, a potential insensitive technique at low bacterial density, as the reference standard for determining diagnostic parameters. Molecular diagnostic techniques were not available to us and our samples were not stored in such a way that these could be applied retrospectively. A further limitation was that BALs were only collected from children annually and therefore the presence of cyanide could only be assessed in terms of its capacity to detect $P$. aeruginosa infections present at the time of the BAL. Finally, compounds present in BAL are highly diluted compared to airway surface liquid. No reliable method to correct for dilution is available. Differences in cyanide concentration between BALs may therefore have reflected, in part, differences in dilution.

In summary, we have shown that although cyanide is detectable in BAL from young children with $\mathrm{CF}$, its measurement has little value in the early detection of $P$. aeruginosa infection. Our results suggest that this may be due, in part, to the association of cyanide concentration with the level of neutrophilic inflammation.

\section{STATEMENT OF INTEREST}

None declared.

\section{ACKNOWLEDGEMENTS}

The complete author list for this manuscript includes the members of AREST-CF listed here: E. Balding, T. Douglas, G.L. Hall, K.M. Logie, C. Murray, S.R. Poreddy, S.M. Stick (all Dept of Respiratory Medicine, Princess Margaret Hospital for Children, Perth, Australia); L.J. Berry, C.L. Gangell, L.W. Garratt, S. Brennan, N. de Klerk, C. Foo, A. Kicic, I.A. Laing, L.S. Mott, E.N. Sutanto, F. Parsons (all Division of Clinical Sciences, Telethon Institute for Child Health Research and Centre for Child Health Research, University of Western Australia, Australia), J.B. Carlin, E. Williamson (Murdoch Children's Research Institute, Victoria, Australia); R. Carzino, J. Harrison, J. Massie, N. Pillarisetti, S.C. Ranganathan, C.F. Robertson, P.J. Robinson, B. Skoric (all Dept of Respiratory Medicine, Royal Children's Hospital, Melbourne, Victoria); P.D. Sly (Division of Clinical Sciences, Telethon Institute for Child Health Research and Centre for Child Health Research, University of Western Australia and Dept of Respiratory Medicine, Princess Margaret Hospital for Children, Perth, Australia) and R. RobinsBrowne (Dept of Microbiology, University of Melbourne, Victoria).

We wish to acknowledge the microbiology departments at Princess Margaret Hospital (Perth, Australia) and the Royal Children's Hospital (Melbourne, Australia) for providing the microbiology data.

\section{REFERENCES}

1 Kosorok MR, Zeng L, West SE, et al. Acceleration of lung disease in children with cystic fibrosis after Pseudomonas aeruginosa acquisition. Pediatr Pulmonol 2001; 32: 277-287.

2 Emerson J, Rosenfeld M, McNamara S, et al. Pseudomonas aeruginosa and other predictors of mortality and morbidity in young children with cystic fibrosis. Pediatr Pulmonol 2002; 34: 91-100.

3 Nixon GM, Armstrong DS, Carzino R, et al. Clinical outcome after early Pseudomonas aeruginosa infection in cystic fibrosis. I Pediatr 2001; 138: 699-704.

4 Douglas TA, Brennan S, Gard S, et al. Acquisition and eradication of $P$. aeruginosa in young children with cystic fibrosis. Eur Respir J 2009; 33: 305-311.

5 Hansen CR, Pressler T, Høiby N. Early aggressive eradication therapy for intermittent Pseudomonas aeruginosa airway colonization in cystic fibrosis patients: 15 years experience. J Cyst Fibros 2008; 7: 523-530.

6 Brennan S, Gangell C, Wainwright C, et al. Disease surveillance using bronchoalveolar lavage. Paediatr Respir Rev 2008; 9: 151-159. 
7 Socransky SS, Gibbons RJ, Dale AC, et al. The microbiota of the gingival crevice area of man. I. Total microscopic and viable counts and counts of specific organisms. Arch Oral Biol 1963; 8: 275-280.

8 Blumer C, Haas D. Mechanism, regulation and ecological role of bacterial cyanide biosynthesis. Arch Microbiol 2000; 173: 170-177.

9 Castric PA. Hydrogen cyanide production by Pseudomonas aeruginosa at reduced oxygen levels. Can J Microbiol 1983; 29: 1344-1349.

10 Worlitzsch D, Tarran R, Ulrich M, et al. Effects of reduced mucus oxygen concentration in airway Pseudomonas infections of cystic fibrosis patients. J Clin Invest 2002; 109: 317-325.

11 Enderby B, Smith D, Carroll W, et al. Hydrogen cyanide as a biomarker for Pseudomonas aeruginosa in the breath of children with cystic fibrosis. Pediatr Pulmonol 2009; 44: 142-147.

12 Ryall B, Davies JC, Wilson R, et al. Pseudomonas aeruginosa, cyanide accumulation and lung function in $\mathrm{CF}$ and non-CF bronchiectasis patients. Eur Respir J 2008; 32: 740-747.

13 Sanderson K, Wescombe L, Kirov SM, et al. Bacterial cyanogenesis occurs in the cystic fibrosis lung. Eur Respir J 2008; 32: 329-333.

14 Sly PD, Brennan S, Gangell C, et al. Lung disease at diagnosis in infants with cystic fibrosis detected by newborn screening. Am J Respir Crit Care Med 2009; 180: 146-152.

15 Australian Respiratory Early Surveillance Team for Cystic Fibrosis. AREST CF. www.arestcf.org Date last updated: August, 8: 2010.
16 Ryall B, Lee X, Zlosnik JE, et al. Bacteria of the Burkholderia cepacia complex are cyanogenic under biofilm and colonial growth conditions. BMC Microbiol 2008; 8: 108.

17 Rosenfeld M, Gibson RL, McNamara S, et al. Early pulmonary infection, inflammation, and clinical outcomes in infants with cystic fibrosis. Pediatr Pulmonol 2001; 32: 356-366.

18 Stelmaszyńska T. Formation of HCN by human phagocytosing neutrophils - 1. Chlorination of Staphylococcus epidermidis as a source of HCN. Int J Biochem 1985; 17: 373-379.

19 Zgliczyński JM, Stelmaszyńska T. Hydrogen cyanide and cyanogen chloride formation by the myeloperoxidase- $\mathrm{H}_{2} \mathrm{O}_{2}-\mathrm{Cl}^{-}$system. Biochim Biophys Acta 1979; 567: 309-314.

20 Stelmaszyńska T. Formation of $\mathrm{HCN}$ and its chlorination to $\mathrm{ClCN}$ by stimulated human neutrophils -2 . Oxidation of thiocyanate as a source of HCN. Int J Biochem 1986; 18: 1107-1114.

21 Pollock JR, Goff HM. Lactoperoxidase-catalyzed oxidation of thiocyanate ion: a carbon-13 nuclear magnetic resonance study of the oxidation products. Biochim Biophys Acta 1992; 1159: 279-285.

22 Wijkstrom-Frei C, El-Chemaly S, Ali-Rachedi R, et al. Lactoperoxidase and human airway host defense. Am J Respir Cell Mol Biol 2003; 29: 206-212.

23 Furtmüller PG, Burner U, Obinger C. Reaction of myeloperoxidase compound I with chloride, bromide, iodide, and thiocyanate. Biochemistry (Mosc) 1998; 37: 17923-17930.

24 Stamyr K, Vaittinen O, Jaakola J, et al. Background levels of hydrogen cyanide in human breath measured by infrared cavity ring down spectroscopy. Biomarkers 2009; 14: 285-291. 